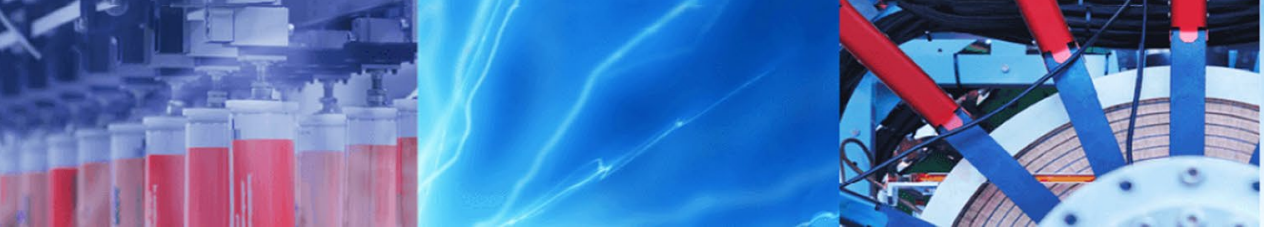

Research Article

\title{
Growth mechanism and motion trajectory of lazy "S" in friction stir welding joint of 6082-T6 aluminum alloy
}

\author{
Hang Zhang ${ }^{1} \cdot$ Yu-meng Sun ${ }^{1} \cdot$ Wen-biao Gong ${ }^{1}\left[\right.$ ] Heng Cui ${ }^{2}$ \\ Received: 30 June 2020 / Accepted: 12 January 2021 / Published online: 3 February 2021 \\ (c) The Author(s) 2021 OPEN
}

\begin{abstract}
Samples of 4-mm-thick 6082-T6 aluminum alloy were subjected to friction stir welding (FSW), and the different lazy "S" morphology of joint cross section and weld surface was obtained corresponding to five groups' welding parameters. Based on the diffusion and kinetics equation of the oxygen-aluminum interface film, a vortex current model of lazy " $\mathrm{S}$ " motion trajectory composed of $(\mathrm{A} \rightarrow \mathrm{B} \rightarrow \mathrm{C} \rightarrow \mathrm{D} \rightarrow \mathrm{E})$ five points which was affected thermomechanical action by temperature field, non-Newtonian fluid and stress field was provided. It was found that the oxygen-aluminum interface film theory not only explained the growth mechanism of lazy " $S$ " and the phenomenon that the lazy "S" on the weld surface disappeared as the welding speed increases, but also clarified the reason why there is no significant difference in cross-section lazy " $\mathrm{S}$ " width. In addition, the vortex current model of the accumulation area at the back of the stirring tool illustrated the regularity of the movement trajectory forming of the cross-section lazy " $\mathrm{S}$ " and the variation of the position of the lazy " $\mathrm{S}$ " of the weld surface deviated from the weld centerline.
\end{abstract}

Keywords Aluminum alloy · Friction stir welding · Lazy "s" · Oxidation kinetics · Vortex model

\section{Introduction}

Friction stir welding (FSW) is a new revolutionary solidstate welding technology [1-4] and has been widely used in aerospace, high-speed rail transit, ship and automobile industries, due to its good mechanical properties of joints, smokeless dust and arc pollution in welding process, less loss, and so on. At the same time, it also has some shortcomings, such as keyhole, hole, lack of penetration, lazy "S," etc. [5]. At present, studies on the lazy "S" are relatively rare. Researchers in the following studies have proposed some research results on the lazy "S."

C. RAJENDRAN et al. [6] found that the tool tilt angle has a significant effect on the weld strength. The welded joint with no hole and tunnel defects can be obtained from tool tilt angle of $1^{\circ}$ to $3^{\circ}$. Okamura et al. [7] reported the initial oxide layer during FSW and its effect on mechanical properties of $6 \mathrm{~N} 01 \mathrm{Al}$ alloy. They suggested that a lazy "S" remained as a vestige of the oxide layer in the cross-sectional stir zone for FSW parameters, the width of the oxide traces found after etching ranges between 10 and $50 \mu \mathrm{m}$ and concluded that the lazy " $\mathrm{S}$ " in the stir zone did not affect mechanical properties of the weld. Wanjara $\mathrm{P}$ et al. [8] determined that with increasing weld pitch, the occurrence of lazy " $S$ " in the weld nugget of friction stir welded AA6061 became increasingly pronounced. A weld pitch of $0.48 \mathrm{~mm} / \mathrm{rev}$ minimizes stir zone softening while limiting the formation of lazy "S." Ren S R et al. [9] compared the butt and stir-in-plate welds and indicated that the lazy " $S$ " did not show up in the welds of stir-in-plate welds and two welds exhibited similar tensile properties and fracture characteristics. Chen huabin et al. [10] and Okamura Hisanori et al. [11] found that there are a large number of spherical or rod-like oxides alongside the lazy "S," and

$\triangle$ Wen-biao Gong, gwbiao@163.com | ${ }^{1}$ Key Laboratory of Advanced Structural Materials of the Ministry of Education, Changchun University of Technology, Changchun 130012, China. ${ }^{2}$ Changchun Railway Vehicles Co Ltd, Changchun 130062 , China. 
they also believed that the lazy "S" was formed because the interfacial oxide layer after being crushed by the stirring pin could not combine with the base metal. Sato et al. [12] studied the lazy " $S$ " in the weld line of 1050 aluminum alloy, indicating that the existence of continuous oxide film is a feature of FSW joint, which is easy to cause cracks. A. Scialpi et al. [13] found that shoulder grooves with different shapes of stirring tool have great influence on clarity and elongation of lazy "S." Wang weibing et al. [14] believe that the lazy " $S$ " is the material demarcation line and there is a cavity behind the stirring tool. Ren et al. [15] made a comparative study of FSW and FSP of 7075Al-T651 and clearly pointed out that the oxide layer of the initial butt surface led to the formation of the lazy "S" and pointed out that removing the surface oxide layer before welding cannot completely eliminate weld joint for lazy "S."Thomas [16] designed three-groove stirring tool and found that the oxide film on the joint surface can be better destroyed by the stir tool during the welding process of the lap joint, promoting the full flow of weld metal and improving the mechanical properties of the joints. Xie tengfei et al. [17] used different pin-shaped stirring tools and found that the lazy " $\mathrm{S}$ " is more easily formed when using conical smooth surface stirring pin and can reduce the welding speed and improve the fluidity of plasticized metal of the weld. It is observed that the lazy "S" on the joint surface will not disappear, but be discontinuously distributed. At present, the research on lazy "S" mainly focuses on the morphological characteristics under the process parameters and whether improving the metal fluidity of the weld under FSP can eliminate lazy "S." However, lazy "S" cannot be simply eliminated by the change of welding process. In this paper, we compared the microstructure of joints under five groups of welding parameters and illustrate the growth mechanism and movement trajectory variation law of lazy "S."

\section{Experiment}

The material used in this study is 6082-T6 aluminum alloy with a thickness of $4 \mathrm{~mm}$. The surface oxide film is removed by grinding the welded sheet. The welding process was performed on FSW-LM-XL16-2D gantry-type numerical control friction stir welding machine. As shown in Fig. 1, the diameters of the stirring tool shoulder and the length of the stirring pin are $12 \mathrm{~mm}$ and $3.85 \mathrm{~mm}$, respectively; the stir pin is conical threads and milling three sloping planes on the side. The tilt angle of the tool was $2.5^{\circ}$ and the penetration depth of the shoulder was $0.1 \mathrm{~mm}$, and other welding parameters are shown in Table 1.

After welding, the weld was sectioned and prepared using standard metallographic techniques. The specimens were ground with 400 grit papers to 1500 grit

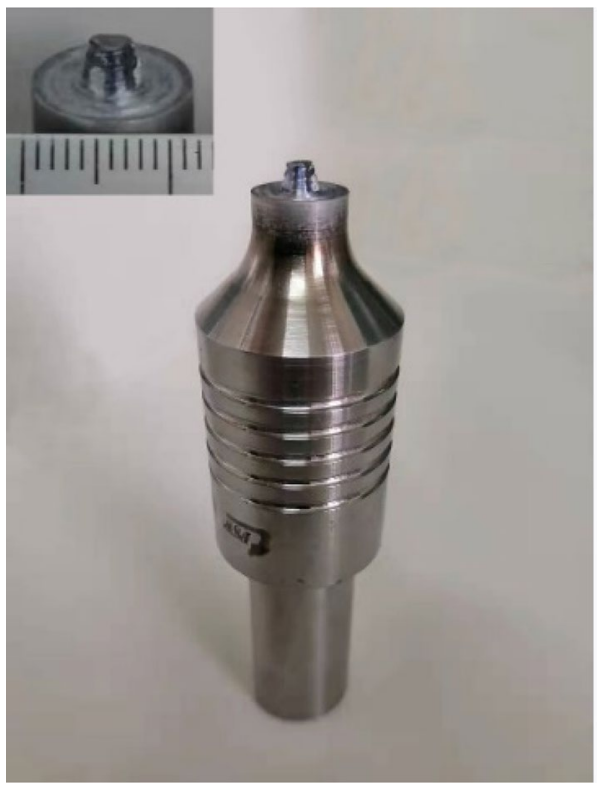

Fig. 1 Schematic diagram of stirring tool

Table 1 Welding parameters

\begin{tabular}{lcll}
\hline $\begin{array}{l}\text { Welding speed } \\
\text { Rotating speed }\end{array}$ & Low Level & Medium level & Higher level \\
\hline $1200 \mathrm{r} / \mathrm{min}$ & $50 \mathrm{~mm} / \mathrm{min}$ & $400 \mathrm{~mm} / \mathrm{min}$ & $1500 \mathrm{~mm} / \mathrm{min}$ \\
& & $500 \mathrm{~mm} / \mathrm{min}$ & \\
& & $600 \mathrm{~mm} / \mathrm{min}$ & \\
\hline
\end{tabular}

papers, polished by high-speed polishing machine, electropolished by $15 \%$ perchloric acid solution and etched by $20 \% \mathrm{NaOH}$ solution. The microstructures of the weld were observed by optical microscopy EPIPHOT-300 (OM, Nikon). The microstructure was analyzed by SEM (Gemini Supra 40).

\section{Results and discussion}

\subsection{Macroscopic metallographic of joints}

Figure 2 shows the cross-section metallographic morphology of different welding parameters after corrosion. The WNZ zone in Fig. 2 (a) (e) presents the unique morphology of friction stir welding: there a black winding line can be seen, which is called lazy " $S$ " by scholars, and there are holes in the shape of square blocks at the bottom of the weld as shown in Fig. 2e. The difference in metallographic morphology of cross section can be observed obviously with the increase in welding speed, the decrease in effective heat input and the increase in 


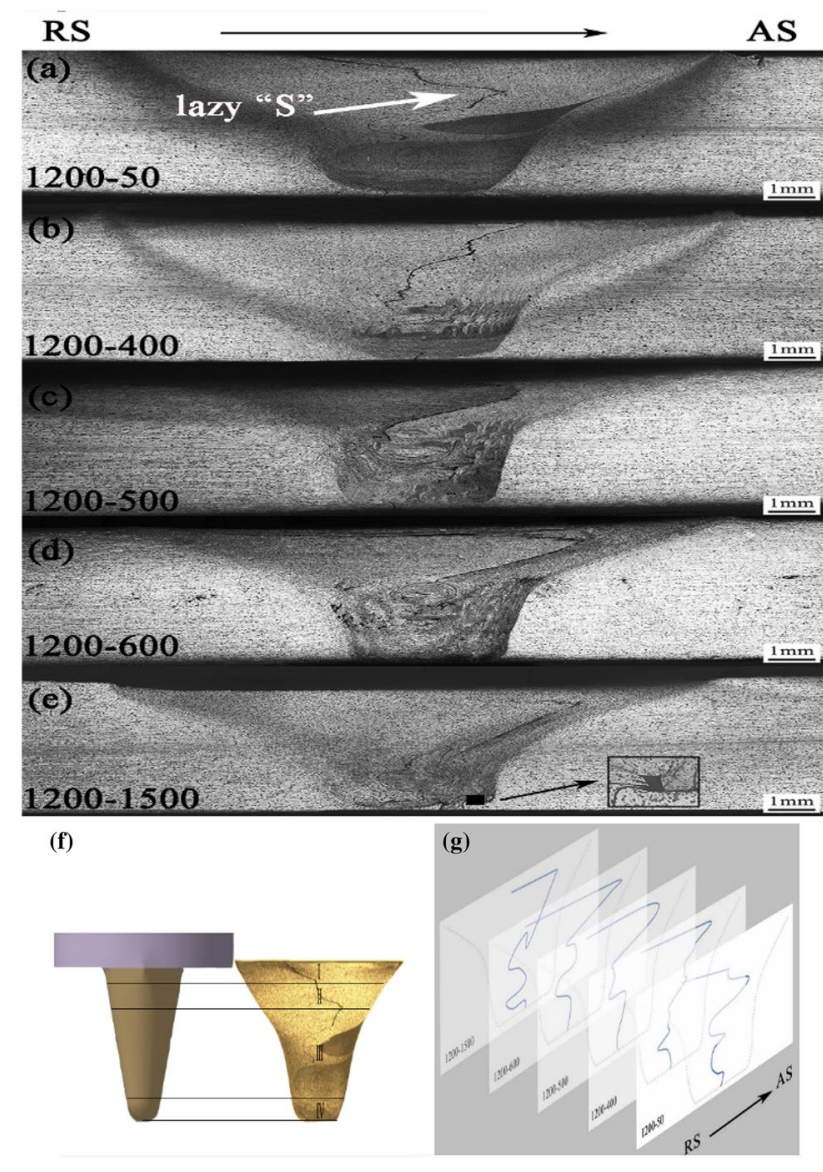

Fig. 2 Metallographic micromorphology of cross section at different welding speeds: (a) 1200-50, (b) 1200-400, (c) 1200-500, (d) 1200-600, (e) 1200-1500, (f) WNZ lazy "S" zone map, (g) The lazy "S" motion trajectory diagram

cooling time of specimen. The bottom width of WNZ riverbed is obviously larger than other welding parameters, and the boundary between TMAZ zone and HAZ zone is the shape of convex. Also, the shape tends to be inverted hill due to the excessive heat flux and metal plastic flow. When the welding speed is $400 \mathrm{~mm} / \mathrm{min}$ in Fig. $2 b$, the bottom width of riverbed becomes narrower, but TMAZ zone is still convex. In Fig. 2c, $d$ and e, TMAZ does not appear in the shape of convex, which is a macroscopic phenomenon caused by the change of mechanical action stirring tool. When the welding speeds are $500 \mathrm{~mm} / \mathrm{min}$ and $600 \mathrm{~mm} / \mathrm{min}$, the cross section is the standard riverbed joint morphology and the corresponding mechanical properties are excellent. From the macrometallographic point of view, there will be hole defects due to the inadequate metal plastic flow when the welding speed is too high and when the welding speed is too low and excessive metal plastic flow will make the shape of the weld tend to hill shape expending the TMAZ and HAZ area, Therefore, unreasonable welding speed parameters should be avoided in engineering application.

Figure $2 \mathrm{f}$ shows the welding joint which was influenced by stirring tool is divided into four areas; the areas from zone I to zone IV are the influence area of shoulder, the influence area of stirring pin, the influence area of stirring pin and the influence area of the bottom of stirring pin, respectively. The schematic diagram of lazy " $\mathrm{S}$ " motion trajectory under five groups of parameters is shown in Fig. 2g. At the same place, all of lazy "S" starts from RS side of the upper end face and grows to AS side, forming sharp angle on AS side and extending toward the center area to the bottom of the weld, and the difference is that the lazy "S" has a large tilt angle at a low welding speed and we cannot find the inflection point at AS side. The turning point affected by stirring pin appeared in area II due to the low welding speed; as for other parameters, the lazy "S" located at the AS side in area I. At medium horizontal welding speed, the lazy " $\mathrm{S}$ " in zone II grows from the first inflection point to the center of WNZ and has a large inclination angle. At high horizontal welding speed, the first inflection point crosses the center of WNZ through zone II and extends to zone III. Zone III is only affected by stirring pin, the morphology of lazy " $\mathrm{S}$ " under five parameters in this zone is different and they all surround the centerline of the weld, because the temperature of zone III during welding is about $465^{\circ} \mathrm{C}$ [18] lower than zone I which is $536^{\circ} \mathrm{C}$ [19]; therefore, the plastic flow of Al matrix is slow and uniform. The matrix material of the end of the stirring pin is subjected to downward extrusion in zone IV and the heat loss adjacent to the lining plate is very high, which leads to the insensitivity of matrix material flow at the bottom of WNZ and the lazy " $S$ " is perpendicular to the bottom line. If the parameters are not selected properly, the lazy "S" will develop into an impermeable defect at the bottom.

\subsection{Lazy "S" on the weld surface}

Figure 3 shows the metallographic morphology of lazy " $\mathrm{S}$ " on the weld surface with different welding speeds. As shown in Fig. 3a, the welding speed is low, the width of the lazy "S" was measured at $3.56 \mathrm{~mm}$, the shape of lazy " $S$ " is like a cloud strip and the edge extends radially with needles, and the cloud strip is located on the RS side. As shown in Fig. 3b, when the welding speed is $400 \mathrm{~mm} /$ $\min \sim 600 \mathrm{~mm} / \mathrm{min}$, the width of the lazy "S" was measured at $2.13 \mathrm{~mm}$, the lazy " $\mathrm{S}$ " is irregular wavy and the edge extends to both sides of the matrix in a needle shape. The edge is not radial and the wavy line is located on the RS side. Figure $3 c$ shows that the width of lazy " $\mathrm{S}$ " is $0.42 \mathrm{~mm}$, the macroscopic lazy " $S$ " is lath and the needle-like edge disappears. Similarly, the lath-like lazy " $S$ " is located on the RS side. Figure $3 d$ shows that the width of lazy " $\mathrm{S}$ " is 

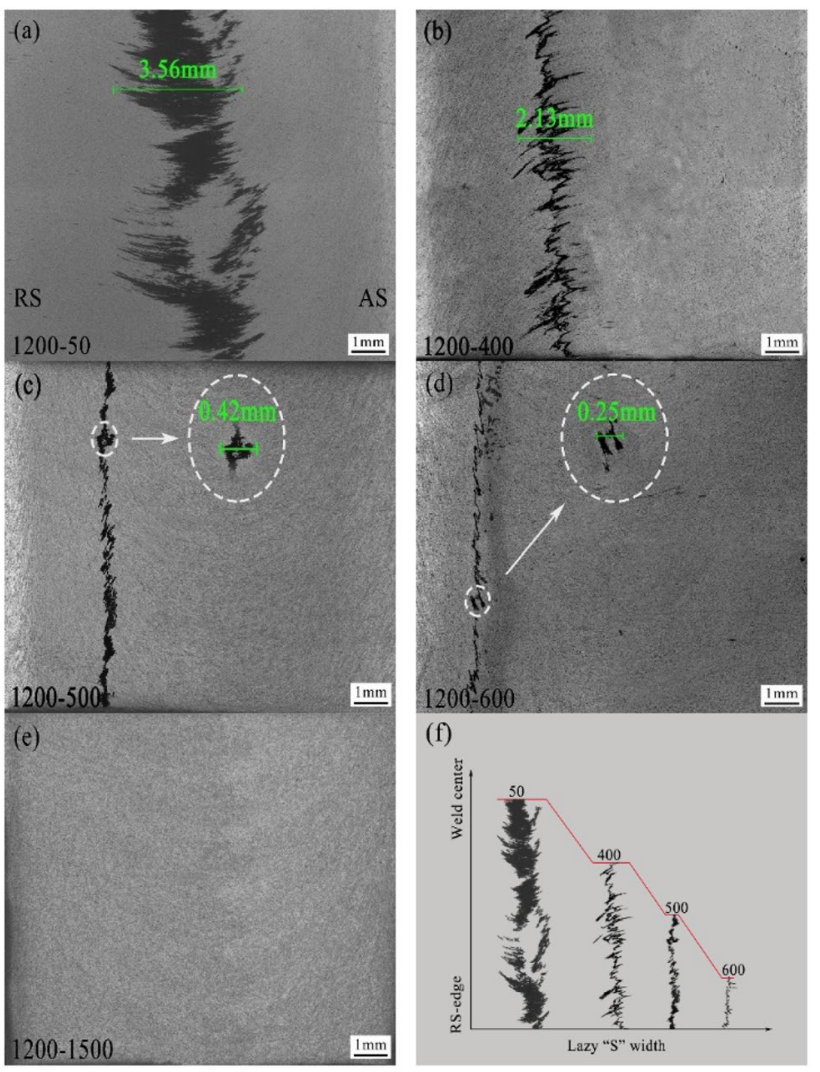

Fig. 3 Metallographic morphology of weld surface at different welding speeds: (a) 1200-50, (b) 1200-400, (c) 1200-500, (d) 1200600 , (e) 1200-1500, (f) schematic diagram of the regularity of weld surface lazy "S"

only $0.25 \mathrm{~mm}$, and the macroscopic observation shows a lighting-like zigzag line with smooth edge transition which is located on the RS side. When the welding speed is high, the macromorphology is smooth and lazy " $\mathrm{S}$ " has not been observed as shown in Fig. 3e.
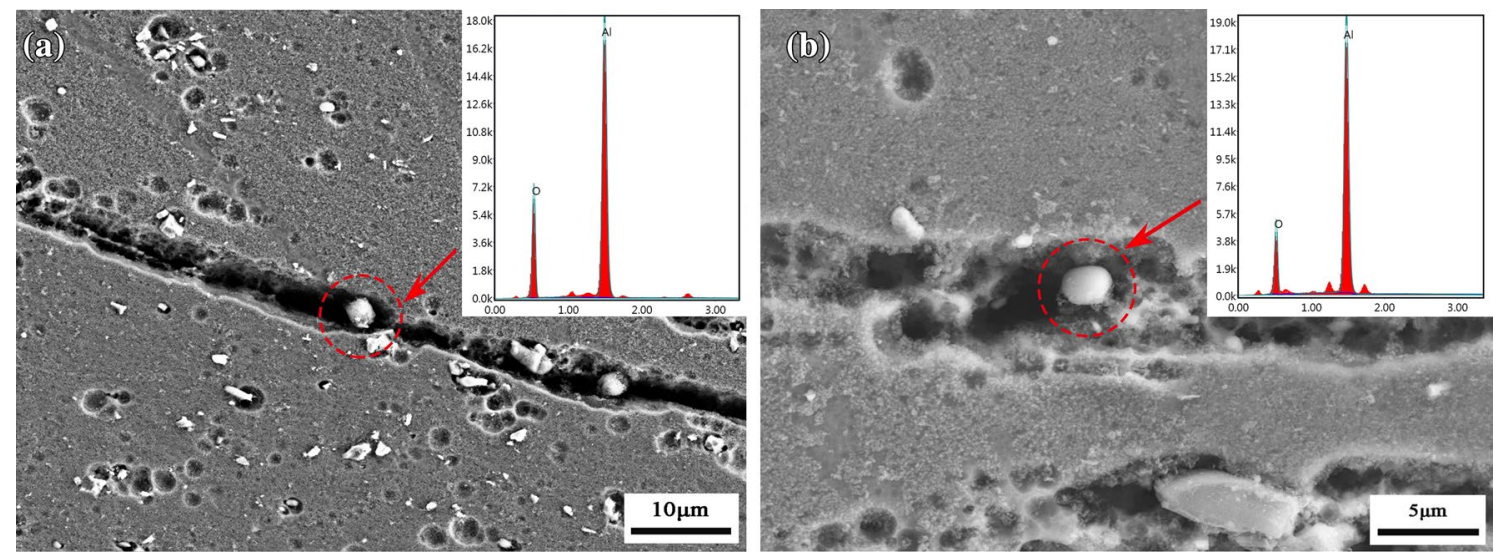

Figure $3 \mathrm{f}$ is a schematic diagram of the regularity of weld surface lazy "S." The morphology of lazy "S" changed with the change in welding speed; when the welding speed is low, the matrix material near the shoulder is viscous, the flow resistance is small and relative sliding is easier. At the same time, the centrifugal force generated by the rotation of the shoulder throws the quasi-liquid aluminum matrix to the edge, which causes the edge of lazy "S" to be elongated into a needle-tip shape; at medium welding speed, the edge burr gradually decreases and eventually tends to be rounded. The horizontal coordinate platform is lazy " $\mathrm{S}$ " width. It is found that the line width along the axis direction is shortened in turn and the lazy " $\mathrm{S}$ " width of welding speed of $600 \mathrm{~mm} / \mathrm{min}$ is shortened by more than $1 / 10$ compared with that of $50 \mathrm{~mm} / \mathrm{min}^{\mathrm{s}}$. The height of the vertical coordinate platform indicates that the lazy "S" deviates from the position relationship of the RS side edge. It can be observed that the platform height decreases with the increase in the welding speed, which means that the lazy "S" gradually moves away from the center of the weld to the RS side.

\subsection{Micromorphology of lazy "S"}

The welding speed is $600 \mathrm{~mm} / \mathrm{min}$ and $1500 \mathrm{~mm} / \mathrm{min}$, and the local micromorphology of lazy "S" is shown in Fig. 4. It was found that the morphology of lazy "S" was similar to that of groove and spherical particles remained in groove. Y. Tao [20] et al. And Q. L. Dai [21] found that $\mathrm{Al}_{2} \mathrm{O}_{3}$ particles remained in and around the lazy "S" by EDS analysis. EDS method was used to analyze the spherical particles marked by red circle in Fig. $4 a$ and b, which were chemical composition consisting of $\mathrm{Al}$ and $\mathrm{O}$, and it can be inferred that the material composition was $\mathrm{Al}_{2} \mathrm{O}_{3}$. It is further proved that lazy "S" is formed in clusters of $\mathrm{Al}_{2} \mathrm{O}_{3}$ particles. Z. ZHANG et al. [22] believed that the morphology of lazy "S" was

Fig. 4 SEM morphology of lazy "S": (a) 1200-600, (b) 1200-1500

\section{SN Applied Sciences}


Fig. 5 a) Chemical diffusion diagram of $\mathrm{Al}_{2} \mathrm{O}_{3}$, (b) $\mathrm{Al}_{2} \mathrm{O}_{3}$ layered structure model

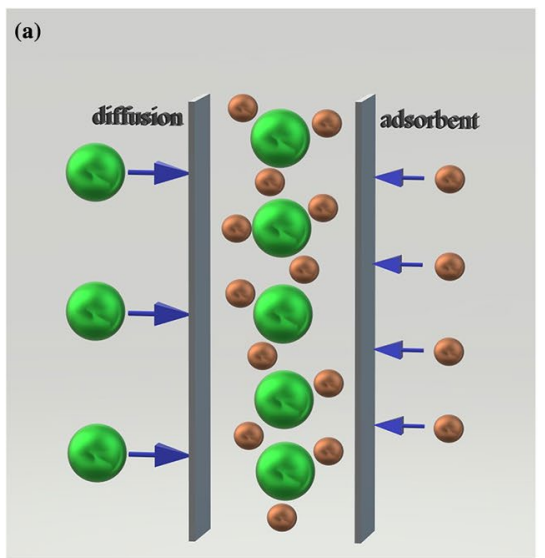

related to welding parameters and the lazy " $S$ " became more and more complete and clear with the increase in welding speed. The width of the lazy " $\mathrm{S}$ " depends on the size range and distribution density of $\mathrm{Al}_{2} \mathrm{O}_{3}$ particles, so the oxidation film on the surface of the workpiece is an important factor affecting the morphology of the lazy "S".

\subsection{Oxygen-aluminum interface film}

At present, many scholars generally believe that the cause of formation of lazy " $S$ " can be simply explained by the fact that $\mathrm{Al}_{2} \mathrm{O}_{3}$ accumulates in WNZ with the plastic flow of the matrix metal and $\mathrm{Al}_{2} \mathrm{O}_{3}$ is insoluble in the matrix material, a groove formed through the cross section after corrosion; however, they neglected the influence of the formation mechanism of oxide film on the composition of lazy "S" before and after friction stir welding. Figure 5 a shows that air $\mathrm{O}_{2}$ is chemically adsorbed on the Al plate, changing oxygen atoms into $\mathrm{O}^{2-}$ ionic state, $\mathrm{O}^{2-}$ dissolves in $\mathrm{Al}$ matrix and reacts with interfacial ionized $\mathrm{Al}^{3+}$ in the form of ion by reverse lattice dif fusion: $4 \mathrm{Al}^{3+}+3 \mathrm{O}^{2-}=2 \mathrm{Al}_{2} \mathrm{O}_{3}$. Ionic state $\mathrm{O}^{2-}$ enters the gap of Al crystal structure to form octahedral crystal structure, and $\mathrm{Al}^{3+}$ is in the gap of oxygen ion; ionic-state $\mathrm{Al}^{3+}$ and $\mathrm{O}^{2-}$ grow layer by layer along the main axis direction of [0001], and $\mathrm{O}^{2-}$ along the direction [1-210] and [10-10] forms the crystal plane (11-20); only two-thirds of the gap has $\mathrm{Al}^{3+}$ as shown in Fig. 5b. After polishing the surface of the specimen within

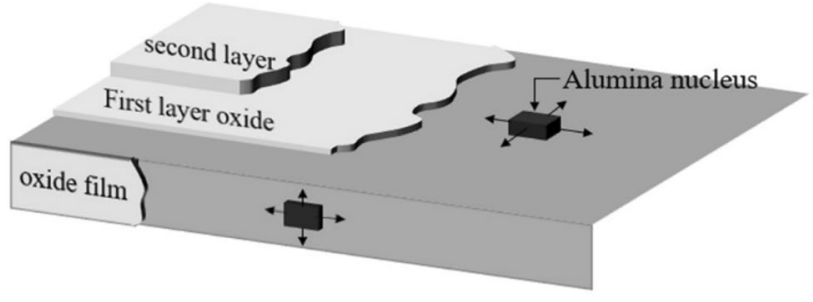

Fig. 6 Oxidation film growth mechanism diagram
$60 \mathrm{~s}$, a single amorphous oxide film will be formed instantaneously and the thickness of oxide film depends on the oxidation rate and diffusion rate of oxide layer. It is found that the thickness of oxide film in natural state extends from $0.5 \mathrm{~nm}$ to $4 \mathrm{~nm}$ [23]

The oxide film has different crystal structure and thickness in natural conditions and during welding, which directly affects the shape and mechanical properties of after welding the lazy "S." Hart [24]and Smeltzer [25]considered that the oxidation kinetics curve was a parabola and the thickness of the oxide film was divided into two levels according to the temperature and the oxide film on the substrate is islandlike and grows layer by layer as shown in Fig. 6. Expression of oxidation reaction rate is:

$r=k\left(\mathrm{Al}^{3+}\right)^{a}\left(\mathrm{O}^{2-}\right)^{b}$

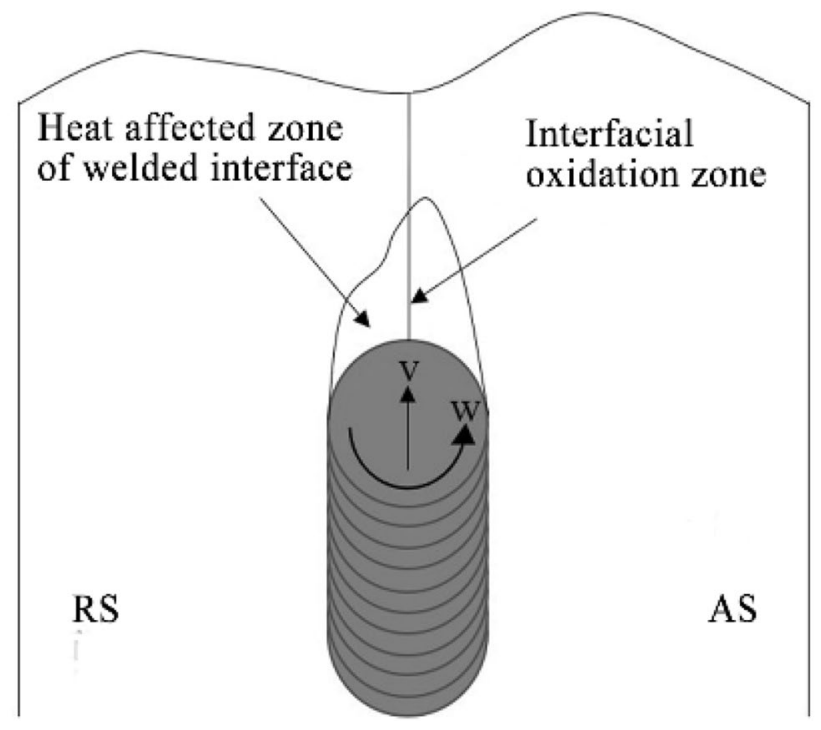

Fig. 7 Welding interface model 
In formula (1), $r$ is the reaction rate; $\mathrm{Al}^{3+}$ and $\mathrm{O}^{2-}$ are the concentration of the reactant; and $a$ and $b$ are the power exponent:

$k=A \exp (-E a / R T)$

In formula (2), $k$ is the rate constant; $A$ is the frequency factor; $R$ is the molar gas constant; $T$ is the thermodynamic temperature; and $E a$ is the activation energy. At room temperature, the concentration gradient driving force of $r / k$ shows a large slope for dynamic curve and the oxidation rate is very fast, which explains the reason why $\mathrm{Al}_{2} \mathrm{O}_{3}$ can be produced instantaneously in a very short time; at the same time, the growth rate of $\mathrm{Al}_{2} \mathrm{O}_{3}$ can be controlled by the electron field. Because the temperature is low and interfacial diffusion driving force is very small, the ability of $\mathrm{Al}^{3+}$ cation to transition outward is limited. Although the concentration of $\mathrm{O}^{2-}$ adsorbed is high, the thickness of oxide film is limited. The interfacial $\mathrm{Al}_{2} \mathrm{O}_{3}$ nuclei grow continuously around the interface, initially forming a thermodynamically stable first layer of amorphous oxide film and the thickness will not increase with the time increasing. Documents [19] have tested the maximum temperature in the nugget zone which reached $536{ }^{\circ} \mathrm{C}$. The temperature rises rapidly, the $r$ on the dynamic curve tends to be straight line, the activation energy of $\mathrm{Al}^{3+}$ atom and the migration ability of $\mathrm{Al}^{3+}$ atom increase, the transition of $\mathrm{Al}^{3+}$ is no longer limited by the electric field force, the growth mechanism is no longer the outward migration of $\mathrm{Al}^{3+}$, but the inward chemical diffusion of adsorbed $\mathrm{O}^{2-}$ along the oxide film interface. Continuous and rapid formation of $\mathrm{Al}^{3+}$ rich second-layer amorphous oxide film was formed on the first layer of oxide film by new $\mathrm{Al}_{2} \mathrm{O}_{3}$ crystal nucleus, and the thickness of the oxide film can

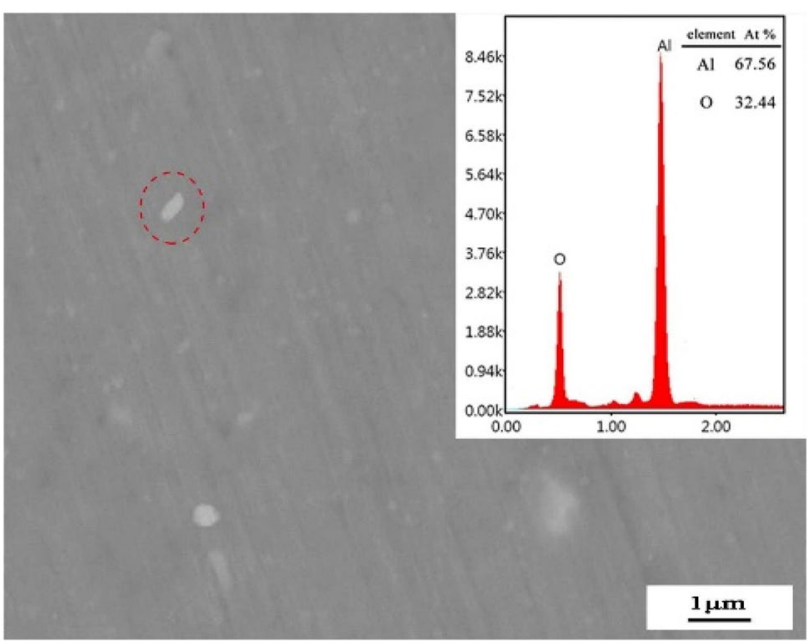

Fig. 8 Distribution diagram of $\mathrm{Al}_{2} \mathrm{O}_{3}$ on weld surface reach $200 \mathrm{~nm}$ [26]. The continuous high temperature during welding transforms the oxide film from amorphous

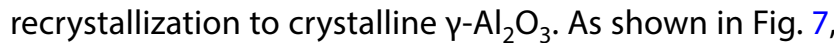
with the continuous welding process, the oxidation film growth is under the condition of high temperature; as the welding speed decreases, the ability to absorb $\mathrm{O}^{2-}$ at the $\mathrm{Al}^{3+}$-rich oxide film interface in the heat-affected zone at the interface to be welded gradually increases and the thickness of the oxide film increases step by step, which explains the phenomenon of the wide difference in the upper surface lazy " $S$ " width in Fig. 2. There is no lazy " $S$ " on the weld surface with the welding speed at $1500 \mathrm{~mm} /$ $\mathrm{min}$; this is due to the fast welding speed, small value of $T$ and $r$ and low oxidation reaction rate. Thus, the thermodynamic ability of the interface absorbed $\mathrm{O}^{2-}$ inward diffusion reduces which leads the second amorphous oxide film to be not easy to grow. The generated oxide film disperses on the weld surface under the mechanical force of the shoulder as shown in Fig. 8. During welding, the interfacial oxidation zone combines tightly and squeezes out excess air from the gap, and according to the oxidation film formation mechanism, the first layer of oxide film is formed in the interfacial oxidation zone at room temperature; however, the interfacial oxidation zone is in a hightemperature state; with the decrease in welding speed, there is not enough $\mathrm{O}^{2-}$ to react with $\mathrm{Al}^{3+}$-rich oxidation film interface to generate oxide film with thickness of hundreds of nanometers, which only transforms to a stable crystalline oxide film. This reasonably explains that there is no difference in the width of lazy " $\mathrm{S}$ " of cross section under different welding speeds.

\subsection{Lazy "S" evolution analysis}

The plastic flow of aluminum matrix material is a kind of vortex phenomenon, which was affected thermomechanical action by temperature field, non-Newtonian fluid and stress field; an understanding vortex model is constructed to explain the motion trajectory of lazy "S" under thermomechanical action, ignoring the influence of stirring tool inclination angle. In Fig. $2 f$, the friction heat generated coulomb model in different areas of temperature field along the thickness direction which is shown as follows:

$Q_{l}=\mu f_{1} \frac{\left(2 \pi r_{1}\right)^{2}}{n}$

In formula (3), $Q$ represents heat flux; $\mu$ is friction coefficient; $f_{1}$ is under-shoulder pressure; $\mathrm{n}$ is rotation speed; and $r_{1}$ is shoulder radius.

$Q_{z}=\int_{r_{2}}^{r_{2}+\tan \alpha L_{z}} \lambda \mu \frac{1}{n} f_{2} r^{2} d r$ 
Fig. 9 a Flow and stress profiles of matrix materials in friction stir welding; (b) lazy "S" trajectory model of vortex state; (c) flowcharts of materials in the cavity on the accumulation side

(a)

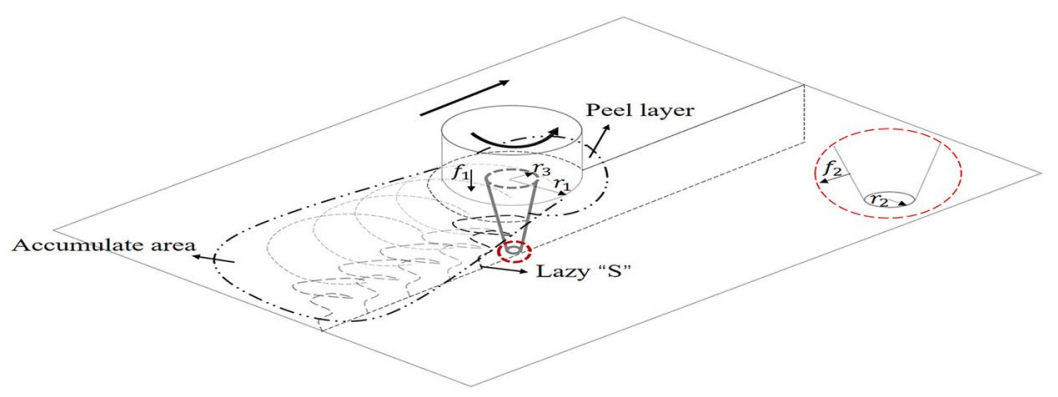

(b)

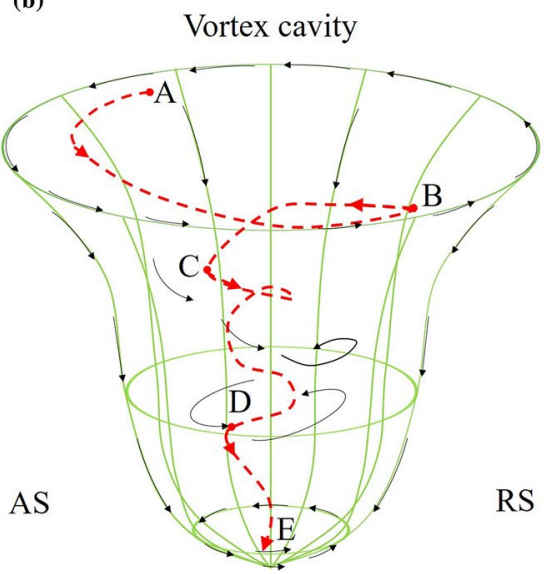

(c)
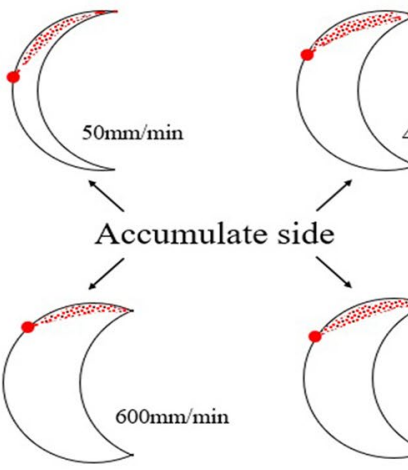

In formula (4), $z$ denotes (II, III, IV); $\lambda$ is a constant; $f_{2}$ is the positive pressure on the end face of the stirring pin; $r_{2}$ is the end radius of the stirring pin; $a$ is the cone angle of the stirring tool; and $L$ is the length of the stirring pin.

Vortex model is generated by temperature field as follows:

$$
J_{n}=\text { Loop Strength/Vortex Strength }=\frac{\iint_{A} \iint v_{n} d s}{\iiint \int \omega_{n} d \sigma}
$$

In formula (5), $J_{n}$ denotes the strength coefficient, $v_{n}$ is the welding rate and $\omega_{n}=2 \pi n$.

Torque force is applied to the boundary of vortex field as:

$\tau_{\mathrm{S}}=2 \pi \mu f_{1} \int_{r_{3}}^{r_{y}} r^{2} d r$

$\tau_{\mathrm{SAZ}}=2 \pi \mu\left(f_{1}+f_{2} \cos \alpha\right) \int_{r_{x}}^{r_{y}} r^{2} d r$

$\tau_{\mathrm{p}}=2 \pi \mu f_{2} \cos \alpha \int_{r_{x}}^{r_{y}} r^{2} d r$ $\tau_{\mathrm{v}}=2 \pi \mu f_{2} \sin \alpha \int_{r_{2}}^{r_{y}} r^{2} d r$

In formula (6) (9), $\tau_{\mathrm{S}}$ represents the shoulder torque; $\tau_{\mathrm{SAZ}}$ is the (I, II) zone torque; $\tau_{\mathrm{p}}$ is the end-face torque of parallel stirring pin; $\tau_{\mathrm{v}}$ is the end-face torque of vertical stirring pin; and $r_{3}$ is the top radius of stirring pin.

As shown in Fig. 9a, a softened peeling layer is formed at the front of the stirring tool; with the change in welding speed, the peeled matrix material forms an instantaneous cavity at the back of the stirring tool, a step length of the cavity span is $\left(S=K \frac{V_{n}}{n}\right)$ and the matrix material in the peeling layer flows into the step-length cavity. Under the downward force of the stirring tool, the oxide film follows the matrix material to fill the cavity in a vortex mode, as shown in Fig. 9b. With the continuous welding, a large number of filling cavities form an accumulation area at the back of the stirring tool. $A \rightarrow B \rightarrow C \rightarrow D \rightarrow E$ five-point connections form the cross-section morphology of the lazy "S." Firstly, we consider the softening degree of the metal in the temperature field, and it can be seen from formulas (3) and (4) that the high temperature of $Q_{1}$ heat flux near the shoulder area I is about $540^{\circ} \mathrm{C}$, which makes the plastic flow of the aluminum matrix produce a thin viscous flow state and its flow resistance is very small and the stirring tool moves rapidly, $Q_{z}$ gradually decreases along the 
thickness direction, making the matrix material near the shoulder present a viscous flow state and the matrix material far away from the shoulder present a thermoplastic state (flow resistance is large) [27]. Secondly, the boundary torque of different metal vortex layers is different, the $\tau_{\mathrm{S}}$ torque is big in the area I adjacent to the shoulder and the plastic flow is viscous state, which makes the $A B$ section become a downward-inclined straight loop, while the $B$ point is located at the edge of the cavity and far away from the shoulder. With the heat flux decreases, the aluminum matrix transits from viscous flow state to thermoplastic state and the flow resistance rises linearly and the $B$ point reaches the maximum. Matrix material flows down to II area, and the shoulder force $f_{1}$ in the formula $\tau_{\text {SAZ }}$ decreases rapidly. Under the effect of vortex strength and $\tau_{\mathrm{v}}$, plastic flow moves to the center of weld, which makes the $B C$ section form $30^{\circ} \sim 45^{\circ}$ downward gyration inclined line. Under the combined action of $\tau_{\mathrm{v}}$ and $\tau_{\mathrm{p}}$, zone III makes the CD segment decrease vertically and the transition arc in this zone comes from $\frac{\tau_{\mathrm{p}}}{\tau_{\mathrm{v}}}>1$; in the region IV at the end of stirring pin, $Q_{z}$ is small in the expression and $\frac{\tau_{\mathrm{p}}}{\tau_{\mathrm{v}}}<1$ and the weld is filled with thermoplastic matrix material which was subjected to a downward force $f_{2}$, which makes the macroscopic appearance of DE segment a vertical line. When the welding speed is low, the heat input is high and the holding time of specimen is long and the vortex layer of viscous flow in SAZ region is thick, making the relative flow easier. The strength coefficient of $J_{50}$ is small and the vortex strength is big, which leads the point $B$ to go down to zone II. When the welding speed is high, the cavity volume formed by step size which makes the plastic flow matrix space too large; under the combined action of loop strength and $\tau_{\mathrm{v}}$, the downward flow velocity of the matrix is too fast and the inclination angle is increased, and it is observed that point C overshoots zone III and the lazy " $\mathrm{S}$ " at point $C$ forms an uphill slope. The oxidized particles crushed by stirring pin move downward along the vortex, which results in the accumulation of $\mathrm{Al}_{2} \mathrm{O}_{3}$ in zones I and $\mathrm{II}$, and the width of the lazy " $\mathrm{S}$ " in zone III becomes narrower due to the gradual increase in flow resistance, which indicates the existence of vortex. The vortex strength and $\tau_{\mathrm{S}}$ on the upper surface are unchanged and the total amount of matrix material flowing into the accumulation area at the weld seam of the specimen remains unchanged, while the step length of the cavity increases with the increase in welding speed; because the edge of the cavity is in the thermoplastic state and the internal friction force is large, the matrix material flowing into the accumulation side adheres to the formation layer by layer, as shown in Fig. 9c, which explains the difference of the lazy "S" deviating from the center position on the surface of the specimen with different welding speeds.

\section{Conclusions}

1. All of the cross section of the joint with five groups of welding parameters showed the lazy "S". When the welding speed was $1500 \mathrm{~mm} / \mathrm{min}$, the hole defects appeared at the bottom of the weld.

2. When the welding parameters range from $50 \mathrm{~mm} / \mathrm{min}$ to $600 \mathrm{~mm} / \mathrm{min}$, the lazy "S" was found on the weld surface. Meanwhile, the line width and deviation from the centerline of the weld were changed regularly. But when welding parameters rose to $1500 / \mathrm{mm} / \mathrm{min}$, the lazy "S" on the weld surface disappeared.

3. The diffusion and kinetic equations of the oxygen-aluminum interface film explained the growth mechanism of the lazy " $S$ " in the friction stirs welding of aluminum alloy and the difference in the width of the lazy "S" on the weld surface.

4. The plastic flow of matrix material is a kind of vortex phenomenon, establishment of vortex model that lazy " $S$ " trajectory is composed of a five-point line $\mathrm{A} \rightarrow \mathrm{B} \rightarrow \mathrm{C} \rightarrow \mathrm{D} \rightarrow \mathrm{E}$. The five-point position relationship is analyzed by the thermomechanical effects of temperature field, non-Newtonian fluid and stress field, illustrating the lazy " $\mathrm{S}$ " on macroevolution process and further verifying the existence of vortex phenomenon. Because the edge of the cavity on the accumulation side is in the thermoplastic state and the internal friction force is large, quantitative matrix material is made attached to the corresponding position, which explains the difference in the lazy "S" deviating from the center of the weld surface.

Acknowledgements The authors wish to express sincere thanks to Jilin Province Development and Reform Commission Industrial Technology Research and Development Project (Grant No.2019C046-7) and Changchun Science and Technology Innovation "Double Ten" Project (Grant No.17ss024) for the financial support.

\section{Compliance with ethical standards}

Conflict of interest On behalf of all authors, the corresponding author states that there is no conflict of interest.

Open Access This article is licensed under a Creative Commons Attribution 4.0 International License, which permits use, sharing, adaptation, distribution and reproduction in any medium or format, as long as you give appropriate credit to the original author(s) and the source, provide a link to the Creative Commons licence, and indicate if changes were made. The images or other third party material in this article are included in the article's Creative Commons licence, unless indicated otherwise in a credit line to the material. If material is not included in the article's Creative Commons licence and your intended use is not permitted by statutory regulation or exceeds the permitted use, you will need to obtain permission directly from the copyright holder. To view a copy of this licence, visit http://creativecommons.org/licenses/by/4.0/. 


\section{References}

1. Gite RA, Loharkar PK, Shimpi R (2019) Friction stir welding parameters and application: A review. Materials Today: Proceedings 19(Pt 2):361-365. https://doi.org/10.1016/j.matpr 2019.07.613

2. Sharma N, Khan ZA, Siddiquee AN (2017) Friction stir welding of aluminum to copper-An overview. Transactions of Nonferrous Metals Society of China 27(10):2113-2136. https://doi. org/10.1016/S1003-6326(17)60238-3

3. Raval SK, Judal K (2020) Recent Advances in Dissimilar Friction Stir Welding of Aluminum to Magnesium Alloys. Materials Today: Proceedings. 22:2665-2675

4. Wang G-Q, Zhao Y-H, Tang Y-Y (2020) Research Progress of Bobbin Tool Friction Stir Welding of Aluminum Alloys: A Review. Acta Metallurgica Sinica (English Letters) 33(1):13-29.

5. Threadgill PL, Leonard AJ, Shercliff HR, Withers PJ (2009) Friction stir welding of aluminium alloys. Int Mater Rev 54(2):49-93.

6. Rajendran C, Srinivasan K, Balasubramanian V, Balaji H, Selvaraj $P$ (2019) Effect of tool tilt angle on strength and microstructural characteristics of friction stir welded lap joints of AA2014-T6 aluminum alloy. Transactions of Nonferrous Metals Society of China 29(9):1824-1835. https://doi.org/10.1016/S1003-6326(19)65090 $-9$

7. Okamura H, Aota K, Sakamoto M, Ezumi M, Ikeuchi K (2002) Behavior of oxides during friction stir welding of aluminum alloy and their effect on its mechanical properties. Weld Int 16(4):266-275. https://doi.org/10.1080/09507110209549530

8. Wanjara P, Monsarrat B, Larose S (2013) Gap tolerance allowance and robotic operational window for friction stir butt welding of AA6061. J Mater Process Technol 213(4):631-640. https://doi.org/10.1016/j.jmatprotec.2012.10.010

9. Ren SR, Ma ZY, Chen LQ (2008) Effect of initial butt surface on tensile properties and fracture behavior of friction stir welded Al-Zn-Mg-Cu alloy. Mater Sci Eng, A 479(1-2):293-299. https ://doi.org/10.1016/j.msea.2007.06.047

10. Huabin C, Keng Y, Tao L, Shanben C, Chengyu J, Yong Z (2006) The investigation of typical welding defects for 5456 aluminum alloy friction stir welds. Mater Sci Eng, A 433(1-2):6469. https://doi.org/10.1016/j.msea.2006.06.056

11. Okamura H, Aota K, Sakamoto M, Ezumi M, Ikeuchi K (2001) Behavior of oxide during friction stir welding of aluminum alloy and its influence on mechanical properties. Journal of the Japan Welding Society 3(19):446-456. https://doi. org/10.2207/qjjws.19.446

12. Sato YS, Takauchi H, Park SHC, Kokawa H (2005) Characteristics of the Kissing-bonding Friction Stir welded Al alloy 1050. Mater Sci Eng, A 405(1-2):333-338. https://doi.org/10.1016/j. msea.2005.06.008

13. Scialpi A, De Filippis LAC, Cavaliere P (2007) Influence of shoulder geometry on microstructure mechanical properties of friction stir welded 6082 aluminum alloy. Materials Design 28(4):1124-1129. https://doi.org/10.1016/j.matde s.2006.01.031

14. Wang W, Tong J, Luan G et al (2013) Model for pre-welding interface disappearance of FSW. Transactions of the China Welding Institution 34(6):37-40
15. Ren SR, Ma ZY, Chen LQ (2007) Effect of welding parameters on tensile properties and fracture behavior of friction stir welded Al-Mg-Si alloy. Scripta Mater 56(1):69-72. https:// doi.org/10.1016/j.scriptamat.2006.08.054

16. Thomas WM, Johnson KI, Wiensner CS (2003) Friction stir welding-recent developments in tool and process technologies. Adv Eng Mater 5(7):485-490. https://doi.org/10.1002/ adem.200300355

17. Tengfei X, Li X, Liming Ke, Guohong L, Chunlin D (2008) Influence of Pin Geometry on Formation of Lazy S in Friction Stir Welding. Hot Working Technology. 07:64-66. https://doi. org/10.14158/j.cnki.1001-3814.2008.07.009

18. Li Y, Sun D, Gong W, Liu L (2019) Effects of post weld aging on the microstructure and properties of bobbin tool friction stir-welded 6082-T6 aluminum alloy. International Journal of Minerals Metallurgy and Materials 26(07):849-857. https://doi. org/10.1007/s12613-019-1800-2

19. Ma FJ, Fu DY, Liu Y et al (2020) The Numerical Simulation of Temperature Field in Friction Stir Welding of 7075 Aluminium Alloy[J]. IOP Conference Series: Materials ence and Engineering. 751(1):012081. https://doi.org/10.1088/1757899X/751/1/012081

20. Tao Y, Zhang Z, Ni DR, Wang D, Xiao BL, Ma ZY (2014) Influence of welding parameter on mechanical properties and fracture behavior of friction stir welded $\mathrm{Al}-\mathrm{Mg}-\mathrm{Sc}$ joints. Mater Sci Eng, A 612(9):236-245. https://doi.org/10.1016/j. msea.2014.06.051

21. Dai QL, Wang XY, Hou ZG, Wu JJ, Shi QY (2015) Microcavities accompanying a zigzag line in friction stir welded A6082-T6 alloy joint. Sci Technol Weld Joining 20(1):68-74. https://doi. org/10.1179/1362171814y.0000000256

22. Zhang Z, Xiao BL, Ma ZY (2013) Effect of Segregation of Secondary Phase Particles and "S" Line on Tensile Fracture Behavior of Friction Stir-Welded 2024Al-T351 Joints. Metallurgical and Materials Transactions A 44(9):4081-4097. https://doi.org/10.1007/ s11661-013-1778-8

23. Jeurgens L P H. (2001). On the Initial Oxidation of Aluminum in Oxygen Gas. Applied Sciences, http://resolver.tudelft.nl/uui... (external link)

24. Hart RK (1956) The Oxidation of Aluminum in Dry and Humid Oxygen Atmospheres. Mathematical Physical and Engineering Sciences. 236(1204):68-88

25. Smeltzer WW (1956) Oxidation of Aluminum in the temperature Range of $400^{\circ}-600^{\circ}$. J Electrochem Soc 103(4):209-214. https:// doi.org/10.1149/1.2430279

26. Jeurgens LPH, Sloof WG, Tichelaar FD, Mittemeijer EJ (2002) Growth kinetics and mechanisms of aluminum-oxide films formed by thermal oxidation of aluminum. J Appl Phys 92(3):1649. https://doi.org/10.1063/1.1491591

27. Luo Wu, Wang Z, Wang, \& Li. (2019) Numerical Simulation of Material Flow and Analysis of Welding Characteristics in Friction Stir Welding Process. Metals 9(6):621. https://doi.org/10.3390/ met9060621

Publisher's Note Springer Nature remains neutral with regard to jurisdictional claims in published maps and institutional affiliations. 